\title{
A Lesson from a Rare Cause of Pulmonary Embolism
}

\author{
Luyao Ma MD, Yan Zhu MD, Haoliang Sun MD, Fanghong Li MD, \\ Yongfeng Shao MD, and Shijiang Zhang MD
}

\section{Introduction}

Pulmonary embolism may be a life-threatening condition that needs immediate medical intervention. Early detection plays an important role in improving the survival rate. However, pulmonary embolism is a difficult diagnosis that may be missed because of non-specific clinical presentation. According to guidelines on the diagnosis and management of acute pulmonary embolism, it should be diagnosed by the combination of the clinical presentation, radiological detection, and blood test such as D-dimer. Generally, anticoagulant therapy is the mainstay of treatment in most cases of pulmonary embolism. Massive or submassive pulmonary embolism causing hemodynamic instability is the indication for thrombolysis or embolectomy. ${ }^{1}$ Most commonly, pulmonary embolism is caused by venous thromboembolism; however, other diseases, such as metastatic tumor, septic emboli, and foreign bodies, can mimic pulmonary thromboembolism. Choriocarcinoma is a malignant, trophoblastic tumor characterized by early hematogenous spread to the lungs. Bagshawe and Brooks were the first to document pulmonary embolism attributable to choriocarcinoma. ${ }^{2}$ Because pulmonary metastatic choriocarcinoma can mimic pulmonary thromboembolism, it makes the differential diagnosis very challenging. We report a 24-year-old woman with pulmonary embolism caused by pulmonary metastatic choriocarcinoma.

Drs Ma, Sun, Shao, and Zhang are affiliated with the Division of Cardiothoracic Surgery; and Drs Zhu and Li are affiliated with the Division of Pathology, First Affiliated Hospital, Nanjing Medical University, Nanjing, People's Republic of China.

The authors have disclosed no conflicts of interest.

Correspondence: Shijiang Zhang MD, Division of Cardiothoracic Surgery, First Affiliated Hospital, Nanjing Medical University, 300 Guangzhou Road, Nanjing 210029 People's Republic of China. E-mail: Shijiangzhang@hotmail.com.

DOI: $10.4187 /$ respcare. 02362

\section{Case Summary}

A 24-year-old woman was admitted for extreme dyspnea and cough. She had experienced 3 spontaneous abortions: the last one 1 year before. She had no documented irregularities in her menstrual period. During the past 4 years she had spent over 10 hours a day playing card games. Six months previous she experienced an episode of sudden dyspnea for the first time, and several days later she sought treatment at a local hospital when her symptoms became unbearable. She was diagnosed as having acute pneumonia, based on chest $\mathrm{x}$-ray, in which several small, scattered nodular shadows appeared in both lungs (Fig. 1). Antibiotics were initiated and her condition improved during the antibiotics course.

She was referred to our hospital because of urgent orthopnea. She was afebrile, with a heart rate of 132 beats/ min, breathing frequency of 32 breaths/min, and blood pressure of 96/52 $\mathrm{mm} \mathrm{Hg}$. On supplemental oxygen (3 L/ min via mask), her $\mathrm{P}_{\mathrm{O}_{2}}$ was $50 \mathrm{~mm} \mathrm{Hg}, \mathrm{P}_{\mathrm{CO}_{2}}$ was $26.3 \mathrm{~mm} \mathrm{Hg}$, and $\mathrm{S}_{\mathrm{pO}_{2}}$ was $89 \%$. Emergency contrastenhanced computed tomography showed several scattered nodular shadows in both lungs, and pulmonary embolism occluding the left pulmonary artery (Fig. 2). Echocardiography supported the diagnosis of pulmonary embolism and illustrated severe pulmonary hypertension, up to $105 \mathrm{~mm} \mathrm{Hg}$, along with mild tricuspid regurgitation. Brain natriuretic peptide was $5,683 \mathrm{pg} / \mathrm{mL}$ (normal $0.5-30 \mathrm{pg} / \mathrm{mL}$ ), and creatine kinase-myocardial band was $44 \mathrm{U} / \mathrm{L}$ (normal 0-23 U/L). D-dimer was $270 \mu \mathrm{g} / \mathrm{L}$ (nor$\mathrm{mal}<500 \mu \mathrm{g} / \mathrm{L})$.

The day after her admission, pulmonary angiography via the right femoral vein showed complete occlusion of the left pulmonary artery (Fig. 3). Filling defects were also observed in several parts of the deep vein of right crus, and were thought to be caused by deep venous thrombosis. During the angiography we failed to dissolve the emboli by direct injection of 400,000 IU urokinase. So to prevent progressive pulmonary thrombus a venous filter was placed in the inferior vena cava. After discussion with consulting physicians, the consensus was to perform pulmonary embolectomy. During the procedure, under cardiopulmonary 


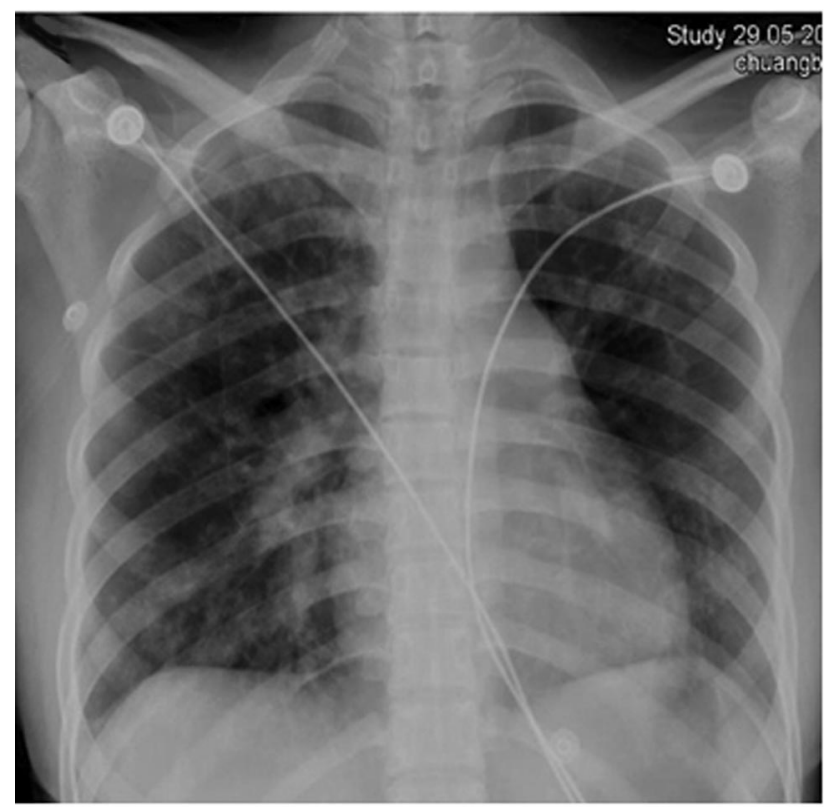

Fig. 1. Chest $\mathrm{x}$-ray shows several small, scattered nodular shadows in both lungs.

bypass, the white and reddish soft emboli (Fig. 4) was found to completely obstruct the opening of the left pulmonary artery and part of the right pulmonary artery. We completely removed the emboli. Prior to decannulation, the pulmonary artery pressure range was $90-110 \mathrm{~mm} \mathrm{Hg}$ and surpassed the systemic pressure. The hemodynamic situation was so desperately unstable that a $5 \mathrm{~mm}$ hole was made in the atrial septum to mitigate the condition. The patient was then transferred to the ICU for monitoring and treatment.

Despite our efforts, the patient died of heart failure due to severe pulmonary hypertension and acute renal failure. To our surprise, pathology found that the pulmonary emboli consisted of intimately related syncytiotrophoblasts and cytotrophoblasts, without formation of definite placental type villi. In conjunction with the positive human chorionic gonadotropin and cytokeratin immunostaining (Fig. 5) in the tumor cells, the diagnosis of choriocarcinoma was confirmed.

\section{Discussion}

Pulmonary embolism is a major cause of mortality and morbidity worldwide. Pulmonary embolism primarily originates from deep venous thrombus, but occasionally other causes have been detected, including metastatic tumor. Pulmonary embolisms from metastatic tumors come mostly from breast cancer, stomach cancer, and lung cancer, whereas choriocarcinoma is rarely found. ${ }^{3}$ As is well known, gestational choriocarcinoma is a highly malignant
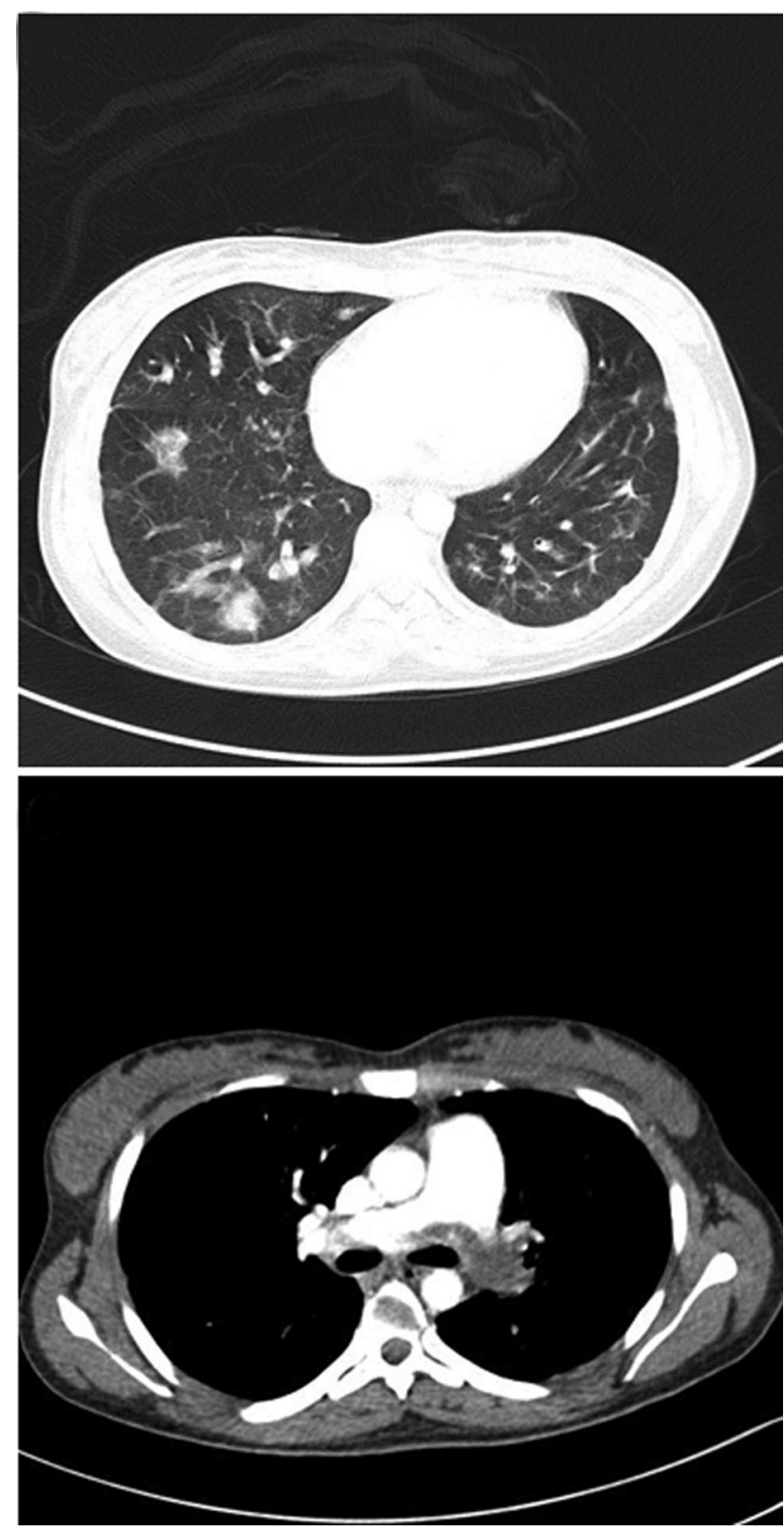

Fig. 2. Contrast-enhanced computed tomography shows (above) scattered nodular shadows in both lungs and (below) occluded left pulmonary artery.

epithelial tumor, arising from the trophoblast of any type of gestational event, about half following a hydatidiform mole, others occurring after abortion (25\%), normal pregnancy $(23 \%)$, or ectopic pregnancy $(2 \%)$. Following Bagshawe and Brooks's first documentation of pulmonary embolism and pulmonary hypertension attributable to choriocarcinoma, ${ }^{2}$ only a few relevant reports have been published. ${ }^{4,5}$

Pulmonary embolism from choriocarcinoma has unique characteristics. ${ }^{4}$ First and foremost, this condition can be completely treated by chemotherapy, even in advanced 


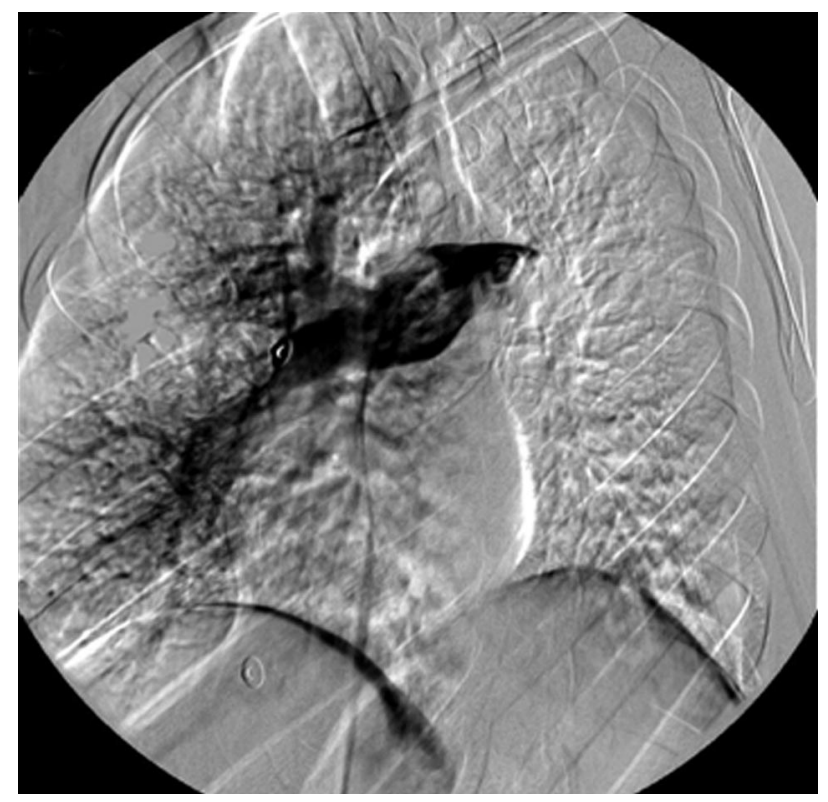

Fig. 3. Pulmonary angiography shows complete occlusion of the left pulmonary artery.

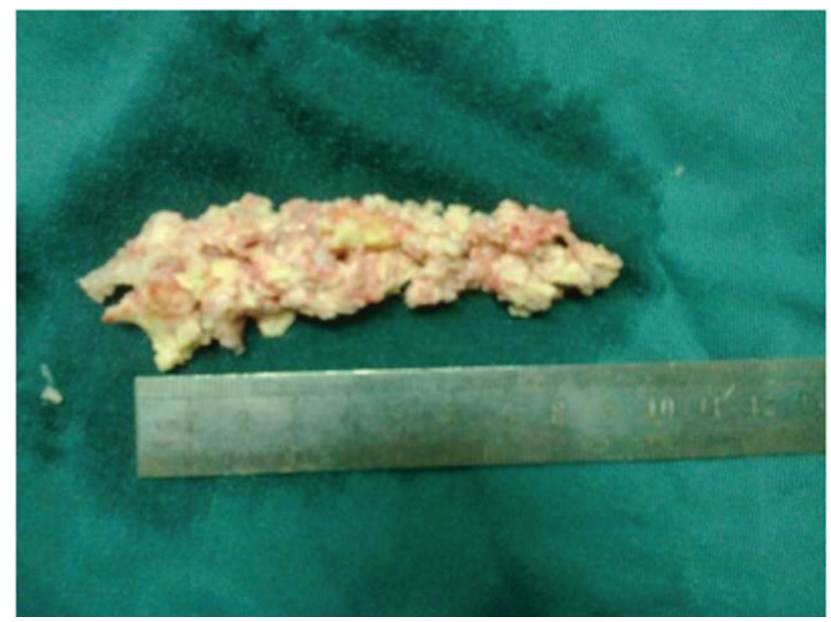

Fig. 4. Multiple fragments of white and reddish soft tissue were removed from the pulmonary artery.

stages. The complete recovery rate of choriocarcinoma is reported to be over $80 \%$ with appropriate chemotherapy. ${ }^{6}$ The second characteristic is remarkably high serum and urinary human chorionic gonadotropin, which is a reliable marker for choriocarcinoma. ${ }^{7}$ In our patient we did not test the serum and urinary human chorionic gonadotropin levels, because we assumed she suffered from pulmonary thromboembolism, based on her history of sedentary behavior and related test results.

Choriocarcinoma is frequently detectable by symptoms of metastatic disease. When the pulmonary metastatic lesions present prior to the primary tumors, a patient with choriocarcinoma is prone to be misdiagnosed as having a
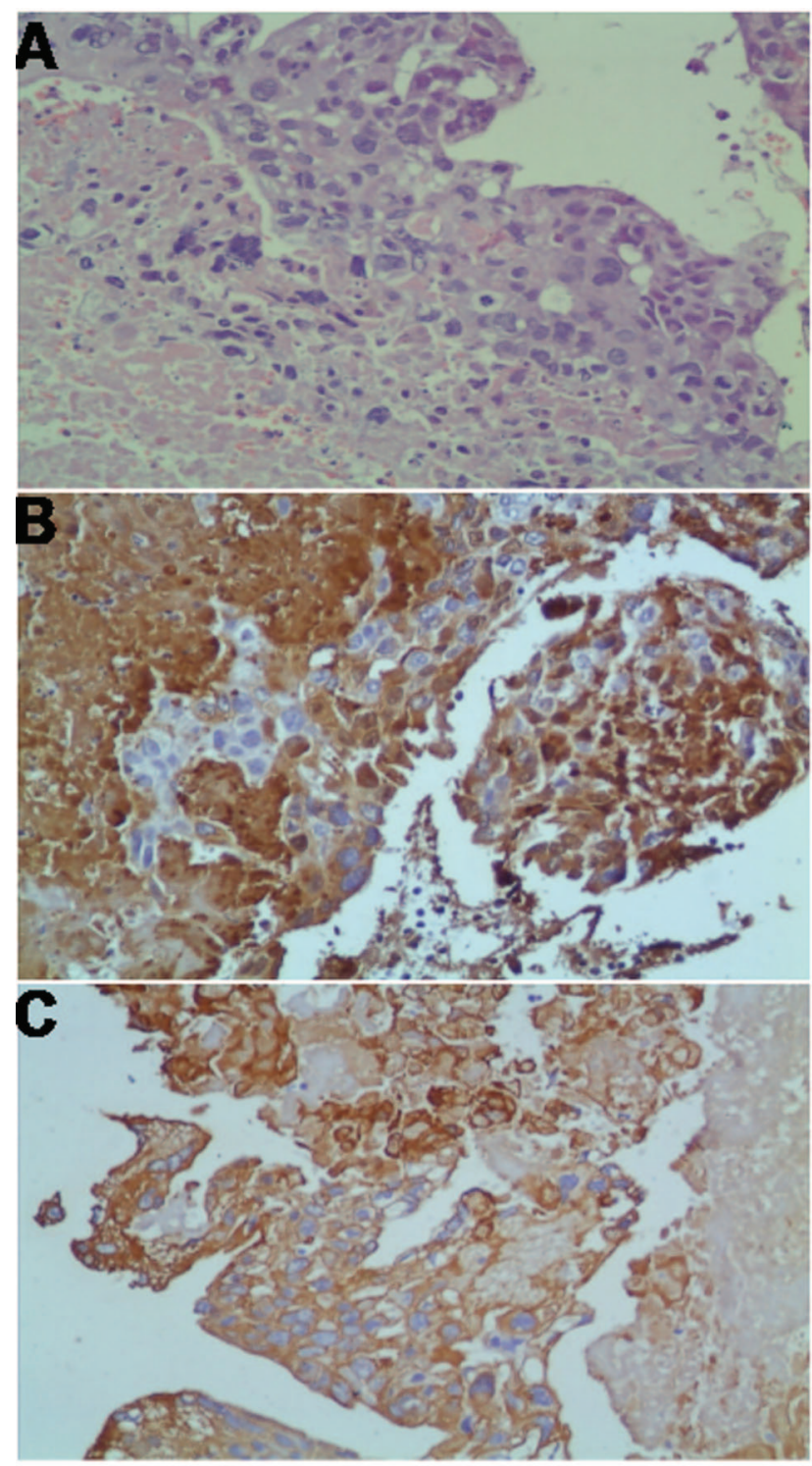

Fig. 5. A: The tumor was constituted by only a thin peripheral rim of viable tissue, with central necrosis and hemorrhage. The tumor cells had an intimate mixture of multinucleated syncytiotrophoblast, mononuleate cytotrophoblast, and intermediate trophoblast. There was considerable cytologic atypia in the trophoblast, with polymorphic- hyperchromatic nuclei and abnormal mitotic figures (hematoxylin-eosin stain, magnification 200). Syncytiotrophoblasts were strongly positive for $(\mathrm{B})$ beta-human chorionic gonadotropin and (C) cytokeratin immunostaining (magnification 200).

pulmonary disease. ${ }^{6}$ In our patient the atypical x-ray findings in both lungs initially misled to the diagnosis of infection; the metastases were difficult to distinguish from nonmalignant lesions. ${ }^{8}$ Although computed tomography and magnetic resonance imaging are valuable in the diagnosis of pulmonary embolism, it is difficult to obtain noninvasive proof of pulmonary embolism of gestational trophoblastic disease. To reduce the risk of misdiagnosis, there is evidence that positron emission tomography can 


\section{A Lesson from a Rare Cause of Pulmonary Embolism}

differentiate tumor embolism from thromboembolism. ${ }^{9}$ Positron emission tomography is very sensitive in identifying malignant tissues, including choriocarcinoma, by their high uptake of fluorodeoxyglucose. Positron emission tomography may be the only noninvasive way to differentiate between a tumor and a blood clot. The other way to diagnosis a tumor embolization is cytology of a pulmonary-artery-catheter-derived blood specimen, which is the most promising new diagnostic technique. ${ }^{10}$

D-dimer, a specific fibrin derivate, is very important in distinguishing between thrombotic and non-thrombotic pulmonary embolism. D-dimer assay is sensitive but not specific, so a positive D-dimer result does not rule in the diagnosis. Pulmonary thromboembolism can almost be excluded safely in patients with a low or moderate clinical probability when the D-dimer is $<500 \mu \mathrm{g} / \mathrm{L} .{ }^{1,11}$ Therefore, when D-dimer is negative, it is reasonable to look for other pulmonary embolism pathogeneses.

\section{Teaching Points}

- The clinical presentation of non-thrombotic pulmonary embolism is nonspecific and similar to that of pulmonary thromboembolism.

- Non-thrombotic causes of pulmonary embolism should be considered in selected patients, according to the patient's entire clinical context, including the possibility of choriocarcinoma, especially in child-bearing women.

- A negative D-dimer in highly sensitive and therefore safely excludes pulmonary thromboembolism in patients with low clinical probability.

- High human chorionic gonadotropin is a reliable tumor marker for choriocarcinoma.

- If possible and needed, positron emission tomography is recommended for differentiating tumor embolism from thromboembolism.

- Pulmonary wedge biopsy is the most promising new diagnostic technique.

- Due to the rarity and high efficacy of chemotherapy for choriocarcinoma and related pulmonary embolism, our primary aim here is to alert physicians to the possibility of pulmonary embolism caused by choriocarcinoma among women of child-bearing age.

- Chemotherapy should be initiated as soon as the diagnosis is made or highly suspected.

- Choriocarcinoma should be considered in the differential diagnosis of fertile women presenting with symptoms of pulmonary embolism or pulmonary hypertension.

\section{ACKNOWLEDGMENTS}

We thank Harrison Stratton, Baiping Cui, Dharshaun Turner, and Darien Lee Allen for their help with English editing.

\section{REFERENCES}

1. Torbicki A, Perrier A, Konstantinides S, Agnelli G, Galie N, Pruszczyk $\mathrm{P}$, et al. Guidelines on the diagnosis and management of acute pulmonary embolism. The task force for the diagnosis and management of acute pulmonary embolism of the European Society of Cardiology (ESC). Eur Heart J 2008;29(18):2276-2315.

2. Bagshawe KD, Brooks WD. Subacute pulmonary hypertension due to chorionepithelioma. Lancet 1959;1(7074):653-658.

3. Roberts KE, Hamele-Bena D, Saqi A, Stein CA, Cole RP. Pulmonary tumor embolism: a review of the literature. Am J Med 2003; 115(3):228-232.

4. Seckl MJ, Rustin GJ, Newlands ES, Gwyther SJ, Bomanji J. Pulmonary embolism, pulmonary hypertension, and choriocarcinoma. Lancet 1991;338(8778):1313-1315.

5. Watanabe S, Shimokawa S, Sakasegawa K, Masuda H, Sakata R, Higashi M. Choriocarcinoma in the pulmonary artery treated with emergency pulmonary embolectomy. Chest 2002;121(2):654-656.

6. Lewis JL Jr. Diagnosis and management of gestational trophoblastic disease. Cancer 1993;71(4 Suppl):1639-1647.

7. Trubenbach J, Pereira PL, Huppert PE, Farnsworth C, Mayer R, Feine U, et al. Primary choriocarcinoma of the pulmonary artery mimicking pulmonary embolism. Br J Radiol 1997;70(836):843-845.

8. Seo JB, Im JG, Goo JM, Chung MJ, Kim MY. Atypical pulmonary metastases: spectrum of radiologic findings. Radiographics 2001; 21(2):403-417

9. Sone T, Yoshikawa K, Fukunaga M. Pulmonary tumor embolism from choriocarcinoma detection with F-18 FDG positron emission tomography. Clin Nucl Med 2008;33(11):773-774.

10. Abati A, Landucci D, Danner RL, Solomon D. Diagnosis of pulmonary microvascular metastases by cytologic evaluation of pulmonary artery catheter-derived blood specimens. Hum Pathol 1994;25(3): 257-262.

11. Kearon C, Ginsberg JS, Douketis J, Turpie AG, Bates SM, Lee AY, et al. An evaluation of D-dimer in the diagnosis of pulmonary embolism - a randomized trial. Ann Intern Med 2006;144(11):812-821. 